\title{
Platelet-rich plasma and simultaneous wound suturing for the treatment of ischial pressure sores
}

\section{Authors:}

Taiju Fujimura, $\mathrm{MD}, \mathrm{PhD}^{1}$;

Shoko Tsujiko, $\mathrm{MD}^{2}$;

Yuki Iwashina, $\mathrm{MD}^{2}$

1 Department of Plastic and

Reconstructive Surgery, Kyoto

Okamoto Memorial Hospital, Kyoto, Japan

2 Department of Plastic and

Reconstructive Surgery, Kyoto First

Red Cross Hospital, Kyoto, Japan

\section{Corresponding author:}

Dr. Taiju Fujimura

Department of Plastic and

Reconstructive Surgery, Kyoto

Okamoto Memorial Hospital,

Kumiyama-cho Sayama

Nishiokuchi 58, Kuze-gun, Kyoto

613-0034, Japan.

Tel.: +81-774-48-5500;

Fax: +81-774-44-5522.

E-mail: taiju@nn.iij4u.or.jp

\section{Author email addresses:}

Taiju Fujimura: taiju@nn.iij4u.or.jp

\begin{abstract}
Ischial sores are caused by a synovial bursa that develops from pressure or shear forces acting on the ischial bone. Because of this mechanism, the actual skin defect of ischial sores is often smaller than the subcutaneous pocket. Therefore, ischial ulcers are frequently treated by complete pocket resection and reconstruction with an appropriate flap. As an alternative method, we here describe the treatment of five patients with relapsing ischial sore by the application of platelet-rich plasma (PRP) and direct closure by suturing. Ischial sores are often found on the buttocks of paraplegia patients who are seated in a wheelchair for long periods, and they recur at a high rate in these individuals. Our method is useful because no normal tissues around the IPS are resected, and thus it is still possible to use local flaps if a sore relapses.
\end{abstract}




\section{Introduction}

Complete pocket resection and flap reconstruction are widely applied for the treatment of ischial pressure sores (IPSs) [1]. However, IPSs have a high incidence of recurrence. When a large flap is created, the surgical scars surrounding the IPS may impede future treatments. In the present study, we therefore evaluated the efficacy of platelet-rich plasma (PRP) and immediate closure via direct suturing. We report the effectiveness of this method in five patients.

\section{Patients and Methods}

\subsection{Patients}

Five patients with a recurrent IPS (age 17-76 years) were selected from the patient population at Kyoto First Red Cross Hospital and Kyoto Okamoto Memorial Hospital during the period from April 2011 to March 2015 for treatment by the application of PRP and direct wound closure via suturing. All five patients had a history of surgical intervention for their IPSs and were informed about the difficulty of making a flap around the IPS. The size of the skin defects ranged from $14 \times 34 \mathrm{~mm}$ to $23 \times 52 \mathrm{~mm}$, and the size of the subcutaneous pockets ranged from $45 \times 70 \mathrm{~mm}$ to $100 \times 110 \mathrm{~mm}$ (Table I) The possibility of direct suturing was checked by drawing the edges of the wounds directly. All ulcers reached the bone layer (National Pressure Ulcer Advisory Panel [NPUAP] Stage 4).

\subsection{Processing the PRP}

The PRP was created immediately before its use. We obtained $50 \mathrm{ml}$ of the patient's peripheral venous blood and collected it into tubes containing an acid-citrate-dextrose solution anticoagulant. The tubes were centrifuged at low speed $(400 \mathrm{~g})$ for $8 \mathrm{~min}$, and the supernatant plasma from each tube was transferred into another tube, which then underwent high-speed centrifugation at $2300 \mathrm{~g}$ for $12 \mathrm{~min}$. After the upper $2 / 3$ to $3 / 4$ of the layer was removed as 
platelet-poor plasma (PPP), the residual plasma was shaken gently and collected as the PRP. The numbers of platelets, red blood cells (RBCs), and white blood cells were counted before and after centrifugation (Table II). No activator for PRP was used.

\subsection{Surgical procedure}

All surgeries were performed under local or lumbar anesthesia. The wound surface was refreshed with scissors or a curette. Autologous PRP was applied to the surface of granulation tissue, and the wound edge was refreshed and then directly closed by double-layer suturing. If the tension of the sutured wound edge seemed too great, a relaxation incision was made. Suction drainage was not used except in one case in which broad undermining was performed to reduce the tension of the wound edge. The patients were advised to not assume a sitting position for 2 weeks following the surgery, during which they were supplied with decompression mattresses for their wheelchair; physical rehabilitation, including push-ups, was also recommended.

\section{Case 1}

Patient 1 was a 75-year-old male with recurrent IPS as a result of limited mobility following a spinal injury. This patient underwent multiple surgeries and an advanced flap reconstruction 1 year before the treatment for IPS, but the sore recurred at the same site (Fig. 1a). Because of an aggravated infection, he was admitted to our hospital. Bedside debridement and irrigation were performed over several days. Just before wound closure, the size of the skin defect was $32 \times 33 \mathrm{~mm}$ and the pocket size was $50 \times 75 \mathrm{~mm}$ (Fig. 1b). A skin incision was made on the border of the pocket, and the wound surface was curetted under direct observation. After sufficient irrigation, PRP was applied to the wound bed. The wound edge was then directly closed. No drainage tube was inserted. We observed 
good adhesion of the wound edge at 1 week after the operation. This patient experienced no recurrence 2 years after surgery (Fig. 1c).

\section{Case 2}

Case 2 was a 62-year-old male with a spinal injury resulting from a traffic accident 40 years earlier. The patient experienced multiple bilateral IPS recurrences. Upon admission to our hospital, he had a $75 \times 22 \mathrm{~mm}$ IPS on his left ischium (Fig. 2a). Initially, we created a V-Y advancement flap from his posterior thigh, but wound dehiscence occurred 1 month later. After several rounds of bedside debridement and irrigation, the wound bed was cleared of infection. Finally, the size of the skin defect was $23 \times 52 \mathrm{~mm}$ and the pocket size was $50 \times 100 \mathrm{~mm}$. We performed curetting of the wound surface, applied PRP, and directly closed the wound (Fig. 2b,c). Good wound adhesion was achieved, and no recurrence was detected at a 1-year follow-up (Fig. 2d).

\section{Case 4}

Case 4 was a 17-year-old paraplegic female with congenital spina bifida. She had undergone two previous surgeries to correct a left-side IPS. She was admitted to our hospital for the treatment of her infectious wound, which was irrigated and debrided daily. After the infectious symptoms subsided, we performed the surgery. The size of the skin defect was $31 \times 42 \mathrm{~mm}$, and the pocket size was $45 \times 70 \mathrm{~mm}$ (Fig. 3a). The wound edge tension was significant because of prolonged inflammation and abundant scarring. Before applying PRP, we made some incisions to relax the skin and then created a rotation flap over the pocket (Fig. 3b,c). Considering the wide dead space, two drainage tubes were inserted and maintained under low suction force (Fig. 3d). No signs of hematoma or seroma were detected postoperatively, and the drainage tubes were removed after 3 days. 
Good wound adhesion was achieved, and no recurrence had been observed as of 2 years after surgery (Fig. 3e).

\section{Discussion}

The ischium and the superior soft tissue support a significant portion of the body's mass when in a sitting position. In patients who spend prolonged periods in the sitting position, especially those with spinal cord injuries, the ischium bears a disproportionate amount of the body's mass for sustained periods and is therefore more susceptible to IPS development [1]. It is well known that the cause of IPSs is a synovial bursa, which develops in response to pressure or shear forces acting on bone. Because of this mechanism, IPSs often have a relatively large pocket compared to the accompanying skin defect [2], which is most often treated via complete pocket resection and reconstruction with an appropriate flap $[3,4]$.

However, because IPSs have high recurrence rates, many other conservative treatment modalities have been developed. One of the most common treatments for IPSs is sclerotherapy via the injection of highly concentrated ethanol to adhere the pocket wall [5]. Other groups have reported the use of basic fibroblast growth factor [6] and negative pressure wound therapy [7]. In this series, we treated five cases of relapsing IPS with the application of PRP and direct wound closing using sutures.

PRP therapy was first reported in 1988 by Marx et al., who applied the process for mandible reconstruction [8]. Since then, PRP therapy has been effectively applied to many types of soft tissue repairs, and there are also many reports of its effectiveness in wound healing $[9,10]$. Platelets contain alpha granules that release several types of cytokines to promote wound healing, vascular synthesis, granulation, and epithelialization.

In the present cases, we did not 
completely remove the bursal surface but could treat the sores appropriately nonetheless. PRP can transform a chronic wound surface into an acute wound surface through the action of transforming growth factor beta (TGF- $\beta$ ) contained in alpha granules that, in turn, activates fibronectin, which subsequently strongly promotes scar adhesion in the bursa [11]. However, if too many RBCs are contained in PRP, the cyst may form a hematoma following wound infection. We therefore confirmed the RBC count of the patient's PRP before and after centrifugation. As shown in Table II, the number of platelets increased by 9 - to 22 -fold, an increase rate that has been reported to be indicative of treatment effectiveness [12]. However, in some cases, the number of RBCs also slightly increased simultaneously.

In our case series, we did not experience an incidence of hematoma. In general, to avoid the development of hematoma, the number of RBCs in PRP should be carefully monitored when PRP is applied to the pockets. In the present cases, we controlled the number of RBCs in PRP through aspiration of the RBC layer after the first centrifugation, but it is difficult to effectively concentrate platelets while reducing the number of RBCs with this method.

We did not use an activator, such as thrombin or calcium chloride, to thicken the activated PRP to a gel. When using PRP in a pocket, as in the present cases, it is not necessary to gel the PRP. In addition, because many inherent activators infiltrate the wound pocket, the need to activate PRP prior to its application is negligible. Indeed, Sandra et al. [13] reported that activated PRP is less effective in wound management because of the decrease of the enhancement of fibroblast differentiation and contractile function.

In two of the presented cases, we added an incision to relax the skin but were careful not to remove too much tissue. The main causative factor for IPSs is maintaining a sitting position in a 
wheelchair for prolonged periods, especially in paraplegic patients. Therefore, surgeons who treat IPSs must take care to reduce the extent of tissue excision, since excision can be problematic in immobile patients. We also routinely reduce the amount of scar tissue and close the wound by direct suturing. Many IPSs have only small skin defects, and most can be closed directly without creating additional tension, although tension on the wound edge can be alleviated by a small relaxation incision and creating a rotation flap.
In conclusion, immobile patients are not good candidates for high-risk surgical procedures under general anesthesia; therefore, the proposed PRP therapy and direct suturing method can be performed under local anesthesia to effectively close IPSs in these patients. Our results showed that this method is useful because it does not require resection of the normal tissues around the wound, and thus these tissues can be reused as local flaps in the event of IPS recurrence. 


\section{Figure Legends}

Figure 1. a: A recurrent IPS of the left ischium. The subcutaneous pocket was relatively large compared to the skin defect. b: After resection, an incision was added to maintain the wound edge. c: The wound as it appeared 3 years after surgery.

Figure 2. a: A recurrent IPS of the left ischium. After posterior thigh flap reconstruction, the wound edge was detached. b,c: PRP was applied in the pocket, and the wound was closed immediately. d: The wound as it appeared 1 year after surgery.

Figure 3. a: A recurrent IPS of the left ischium. b-d: After resection of the scar tissue, the wound edge was maintained. PRP was applied and the wound was closed immediately. e: The wound as it appeared 2 years after surgery. 
(a)
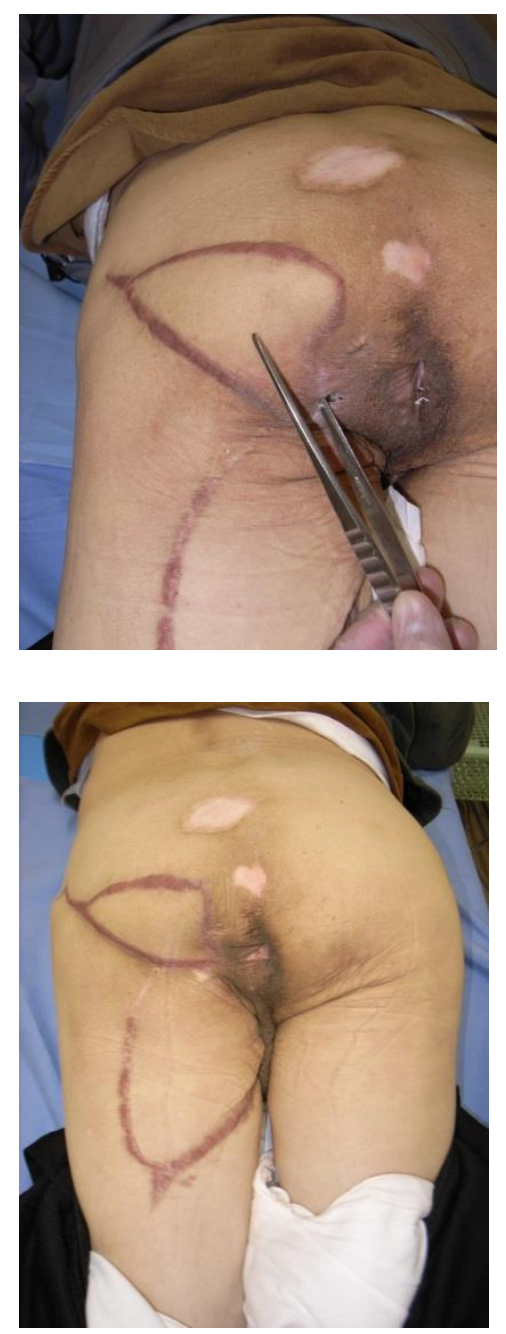

(b)

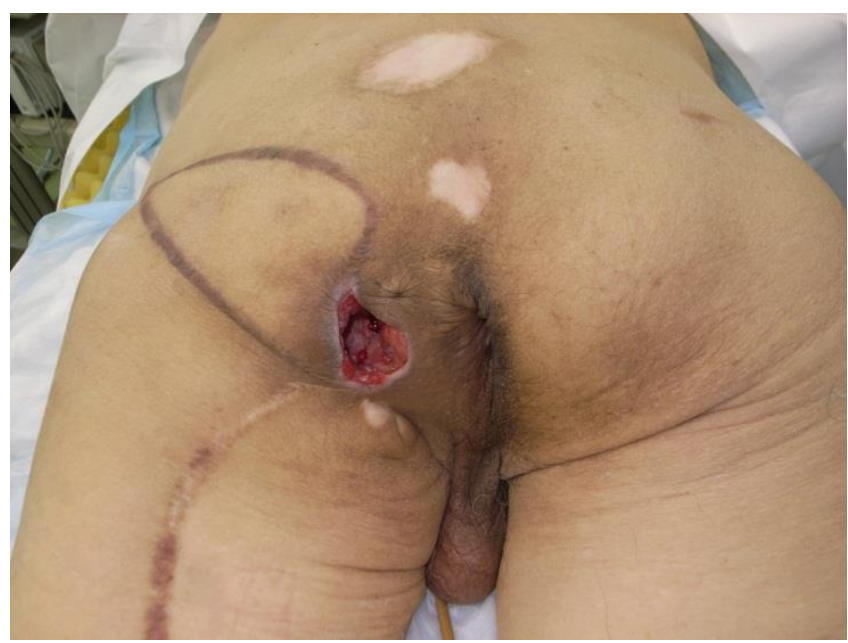

(c)

\section{Fig. 1}


(a)
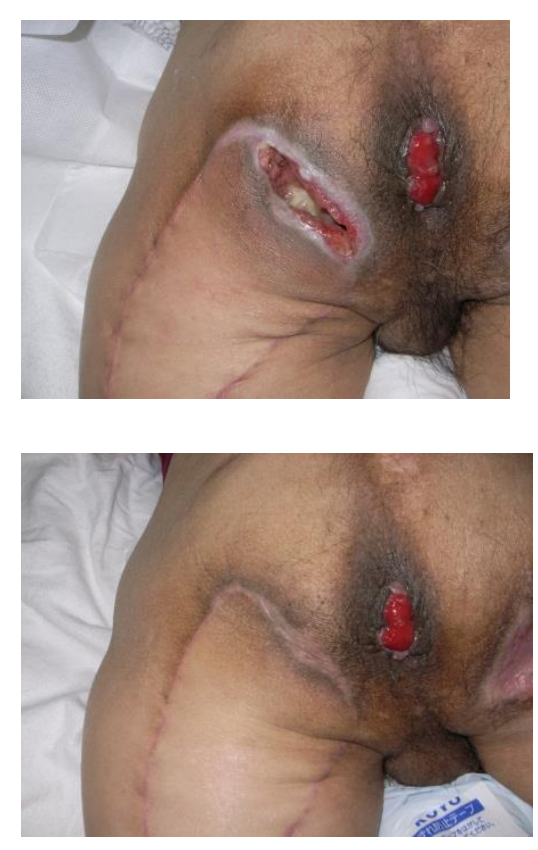

(c) (b)
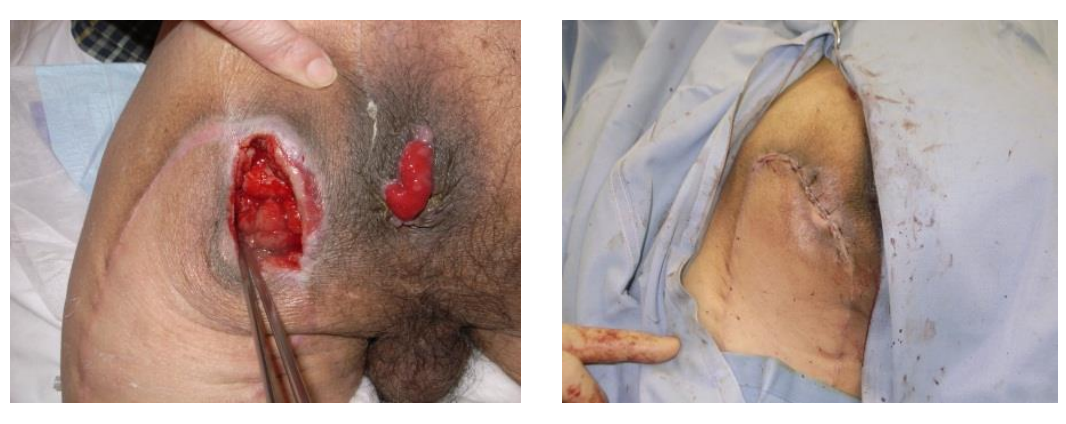

Fig. 2

(d) 
(a)

(b)

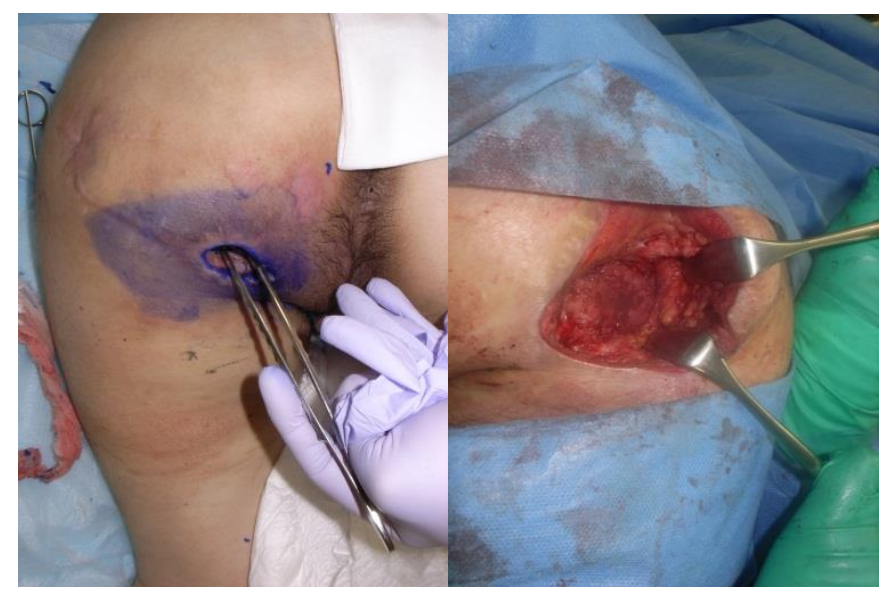

(c)

(d)
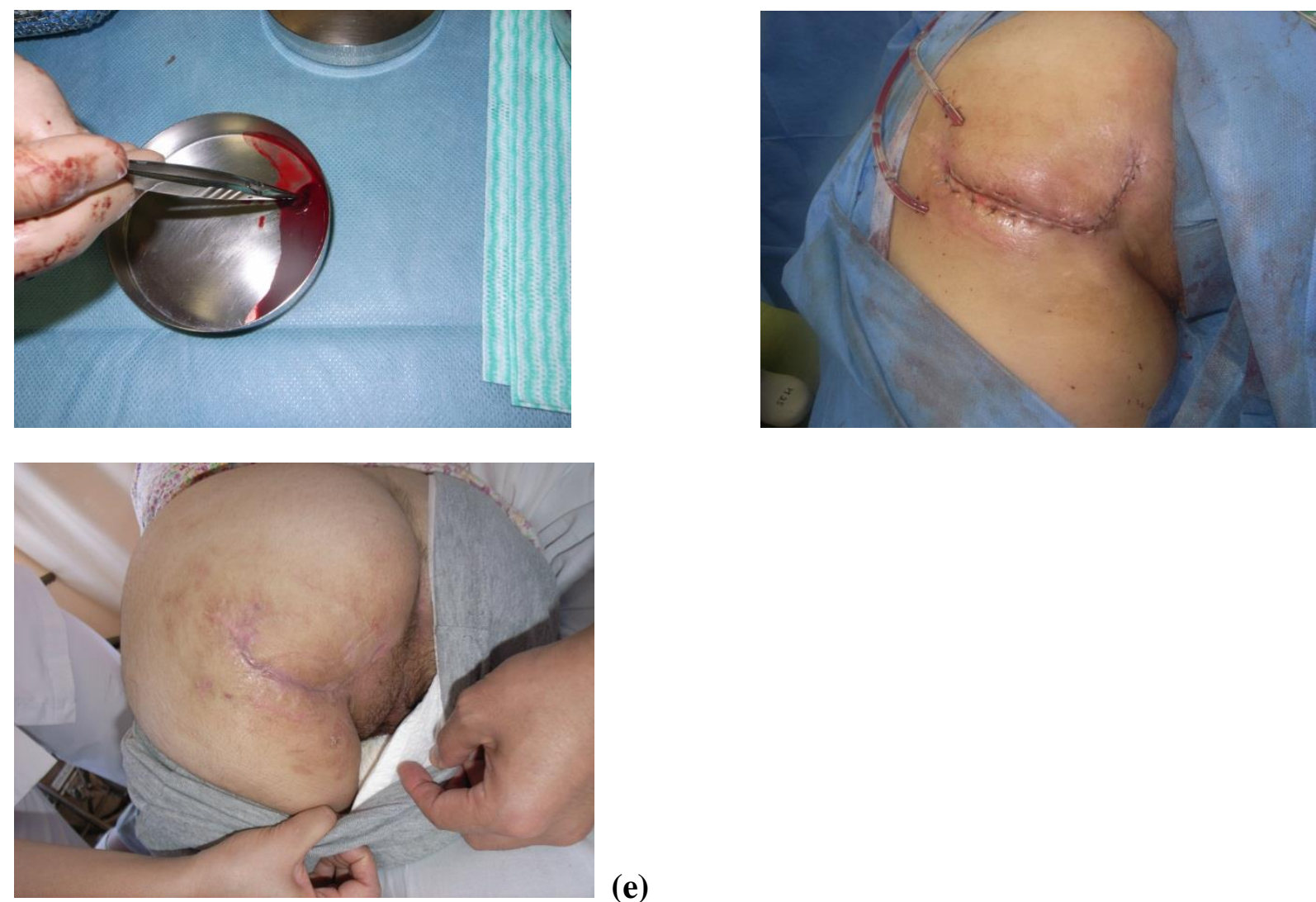

Fig. 3 
Table I. Patient characteristics

\begin{tabular}{|c|c|c|c|c|c|}
\hline $\begin{array}{c}\text { Case } \\
\text { no. }\end{array}$ & $\begin{array}{c}\text { Side of } \\
\text { IPS }\end{array}$ & Age/Gender & $\begin{array}{c}\text { Size of skin } \\
\text { defect } \\
(\mathbf{m m})\end{array}$ & $\begin{array}{c}\text { Size of pocket } \\
(\mathbf{m m})\end{array}$ & Treatment method \\
\hline 1 & left & $76 / \mathrm{M}$ & $12 \times 23$ & $50 \times 75$ & PRP + direct suture \\
\hline 2 & left & $63 / \mathrm{M}$ & $23 \times 52$ & $50 \times 100$ & PRP + direct suture \\
\hline 3 & right & $43 / \mathrm{M}$ & $20 \times 30$ & $100 \times 110$ & $\begin{array}{c}\text { PRP + direct suture } \\
+ \text { relaxing incision }\end{array}$ \\
\hline 4 & left & $17 / \mathrm{F}$ & $31 \times 42$ & $45 \times 70$ & $\begin{array}{c}\text { PRP + direct suture } \\
+ \text { relaxing incision }\end{array}$ \\
\hline 5 & right & $61 / \mathrm{F}$ & $14 \times 34$ & $80 \times 95$ & PRP + direct suture \\
\hline
\end{tabular}


Table II. RBC counts before and after centrifugation.

\begin{tabular}{|c|c|c|c|c|c|c|c|c|c|}
\hline $\begin{array}{c}\text { Case } \\
\text { no. }\end{array}$ & $\begin{array}{c}\text { No. of platelets } \\
\text { before } \\
\text { centrifugation }\end{array}$ & $\begin{array}{c}\text { No. of platelets } \\
\text { after } \\
\text { centrifugation }\end{array}$ & Concentration & $\begin{array}{c}\text { No. of RBCs before } \\
\text { centrifugation }\end{array}$ & $\begin{array}{c}\text { No. of RBCs } \\
\text { after } \\
\text { centrifugation }\end{array}$ & Concentration & $\begin{array}{c}\begin{array}{c}\text { No. of WBCs } \\
\text { before }\end{array} \\
\text { centrifugation }\end{array}$ & $\begin{array}{c}\text { No. of WBCs } \\
\text { after } \\
\text { centrifugation }\end{array}$ & Concentration \\
\hline 1 & 222 & 4045 & 18.22 & 3.88 & 3.80 & 0.98 & 4.97 & 66.6 & 13.4 \\
\hline 2 & 219 & 2037 & 9.30 & 4.85 & 5.42 & 1.12 & 6.21 & 55.3 & 8.90 \\
\hline 3 & 356 & 7812 & 21.9 & 3.93 & 3.22 & 0.82 & 7.31 & 111.6 & 15.3 \\
\hline 4 & 203 & 3184 & 15.7 & 4.28 & 4.45 & 1.04 & 5.94 & 66.2 & 11.14 \\
\hline 5 & 214 & 2536 & 11.85 & 3.67 & 4.32 & 1.18 & 7.1 & 63.8 & 8.99 \\
\hline
\end{tabular}




\section{References}

1. Nakanishi H, Seike T. Characteristics and surgical treatment for ischial pressure sores. Jpn J Plast Reconstr Surg 2003;46:567-75.

2. Homma K, Murakami G, Fujioka H, et al. Treatment of ischial pressure ulcers with a posteromedial thigh fasciocutaneous flap. Plast Reconstr Surg 2001;108:1990-6.

3. Lin $\mathrm{H}$, Hou C, Chen A, et al. Long-term outcome of using posterior-thigh fasciocutaneous flaps for the treatment of ischial pressure sores. J Reconstr Microsurg $2010 ; 26: 355-8$

4. Ahluwalia R, Martin D, Mahoney JL. The operative treatment of pressure wounds: a 10-year experience in flap selection. Int Wound J 2010;7:103-6.

5. Hayashi T. Treatment of ischial pressure sores by means of sclerotherapy using absolute ethanol. Ann Plast Surg 2004;53:554-9.

6. Ono T. Treatment of sacral pressure ulcer with giant pocket utilizing trafermin. Mie Med 2005;49:25-9.

7. Baynham SA, Kohlman P, Katner HP. Treating stage IV pressure ulcers with negative pressure therapy: a case report. Wound Manage 1999;45:28-35.

8. Marx RE, Carlson ER, Eichstaedt RM, et al. Platelet-rich plasma-growth factor enhancement for bone grafts. Oral Surg Oras Med Oral Pathol Oral Radiol Endod 1998;85:638-46.

9. Scott AS, Jeffery JE, Timothy WR, et al. A case report on the use of sustained release platelet-rich plasma for the treatment of chronic pressure ulcers. J Spinal Cord Med 2011;34:122-7.

10. Scimeca CL, Bharara M, Fisher TK, et al. Novel use of platelet-rich plasma to augment curative diabetic foot surgery. J Diabetes Sci Technol 2010;4:1121-6. 
11. Ogawa Y, Sawamura SJ, Ksander GA, et al. Transforming growth factors-beta 1 and beta 2 induce synthesis and accumulation of hyaluronate and chondroitin sulfate in vivo. Growth Factors 1990;3:53-62.

12. Gonshor A. technique for producing platelet-rich plasma and platelet concentrate. Int $\mathrm{J}$ Periodontics
Restorative Dent 2002;22:547-57.

13. Sandra SS, Mickael T, Enrico V. Nonactivated versus thrombin-activated platelets on wound healing and fibroblast to myofibroblast differentiation in vivo and in vitro. Plast Reconstr Surg 2012;129:46e-54e. 